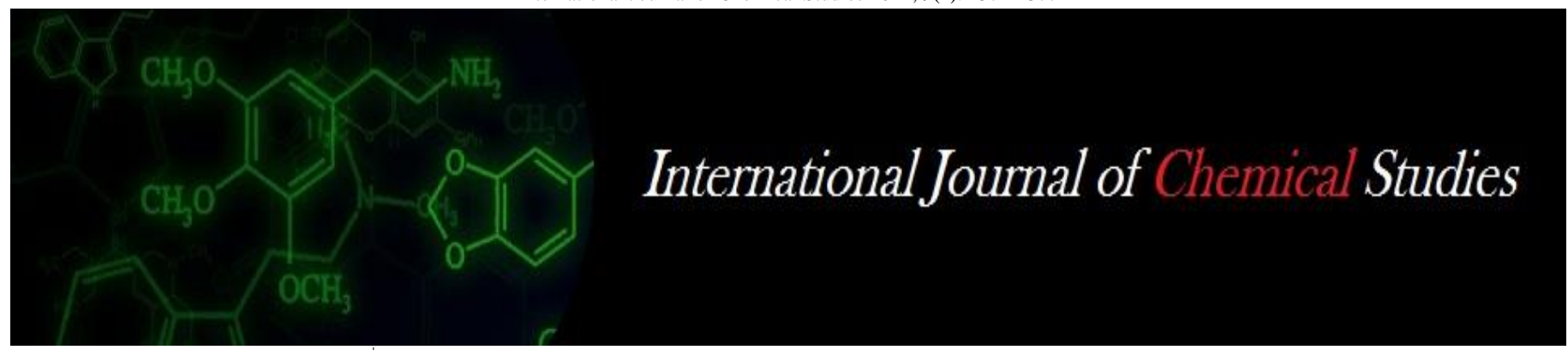

P-ISSN: 2349-8528

E-ISSN: 2321-4902

www.chemijournal.com

IJCS 2021; 9(1): 2374-2377

(C) 2021 IJCS

Received: 05-10-2020

Accepted: 16-12-2020

\section{A Sangeeta}

Indian Institute of Food

Processing Technology,

Department of Food Product

Development, Thanjavur, Tamil

Nadu, India

\section{R Jaganmohan}

Indian Institute of Food

Processing Technology,

Department of Food Product

Development, Thanjavur, Tamil

Nadu, India

\section{Changes in the pasting properties of millet grains due to different processing methods using rapid Visco analyzer}

\author{
A Sangeeta and R Jaganmohan
}

DOI: https://doi.org/10.22271/chemi.2021.v9.i1ag.11584

\begin{abstract}
Millets are considered as under-utilized crops compared with other cereal grains but the year 2018 has been declared as the year of millets after understanding their importance as they are crops that has tendency to fulfill the requirements if the future generations. It possesses unique inherent characteristics of adaptation that makes them sustain harsh climatic changes tolerance which includes resistance to drought, high temperatures, low soil fertility and diseases or pests or for making special foods or beverages because of which they are gaining more attention in the recent years as an alternative to the cereal grains. Millets possess phytochemicals that has capacity to prevent or reduce several diseases thereby imparting several health benefits upon consumption; helps in delaying occurrence of noncommunicable diseases. So facing the climatic changes along with maintaining or improving the health of its nation is the major concern which can be counteracted with the help of "nutri-cereals" which possess unique characteristics with which it can sustain the dramatic climatic changes that is happening or will happen in the future where other fine cereals like paddy and wheat would fail to survive. The present work analyses the effect on pasting properties of millet grains which has given different hydrothermal treatments.
\end{abstract}

Keywords: Kodo millet, rapid Visco analyzer

\section{Introduction}

Cereal grains plays an important role as a major food source in human diet in all parts of the world. The production area and productivity of cereal grains had seen an enormous increase with production area almost doubled in the recent years. Millet presently comprises approximately $11.4 \%$ of the total area employed for cereals and $4.1 \%$ of the total cereals output (Rathore, 2016) ${ }^{[6]}$.

"Miscellaneous crops" or "coarse grains" are the synonym terms used to describe millets. The word millet is derived from a French word "mille" which represents thousands, in context refers to as in a handful take of millets will contain thousands of grains. Millets can be further divided as major millets based on their production capacity consisting of Pearl millet, Foxtail, Proso and then Finger millet and second category as minor millets comprising of kodo, Japanese, barnyard, little millet, fonio and teff millet (Shahidi \& Chandrasekara, 2013) ${ }^{[8]}$. Sorghum and maize were also considered to belong to the millet class in the earlier times class but with the advancement and increased consumption of these crops led to their own separate status as a class from millets. So with the advancement in technologies for their improvement and increased usage other millet crops can also get their separate status in the era (Spengler, 2019) ${ }^{[9]}$.

Kodo millet (Paspalum scrobiculatum L.): since 3000 years ago it has been domesticated in India. Paspalum scrobiculatum grows well in damp habitats across the Old World tropics. It is harvested as a wild cereal in West Africa and in India. Kodo millet is recognized by three races: regularis, irregularis, and variabilis. It is considered as an annual millet with height upto 30 to $90 \mathrm{~cm}$ (de Wet et al., 1983) ${ }^{[1]}$.

Rapid visco analyzer: It is a type of rotational viscometer which measures viscosity of the starch under controlled conditions of temperature and shear (Rapid Visco Analyser, 2000) ${ }^{[5]}$. It is widely used to determine the pasting behavior of flour and starches, it utilizes a heatingcooling cycle to analyze the viscosity of the given starch (Kweon et al., 2010) ${ }^{[2]}$.
Corresponding Author: R Jaganmohan Indian Institute of Food Processing Technology, Department of Food Product Development, Thanjavur, Tamil Nadu, India 
Viscosity is the principal parameter that characterizes the flow properties of fluids. Food Rheology is defined as the study of the deformation and flow of the raw material, intermediate products or final products of the food industry; it finds application in food acceptability, food processing and handling. The vast majority of cereal-based food matrices are systems resulting from cooking starch in excess water. Moreover, they could also be composed of hydrocolloids, fibers and proteins. Therefore, the study of the rheology during heating-cooling cycles finds an important application. In order to analyze the rheology in such conditions, the combination of a container capable of stirring the ingredients under controlled temperature, the possibility of controlling the temperature and the heating-cooling rate as well as the applied shear stress could turn a viscometer into a powerful and versatile tool to elucidate viscosity indicators. Several specific examples of elucidating and characterizing (Kweon et al., 2010) ${ }^{[2]}$. RVA breakdown is caused by disruption of the gelatinized starch granule structure. Thus, the extent of breakdown is caused by the difference between the viscosity when swollen, gelatinized starch granules existed and the viscosity when the gelatinized starch granules are disrupted, either partially or completely. The cooking behaviour of starches and the viscosity of the resulting pastes can be studied with an instrument called Rapid Visco Analyzer (RVA). The RVA profile is generally used as one of the indirect indicators for eating quality in rice sensory evaluation. Viscosities at the start of the holding period and during cooling reflect the ease of cooking starch and paste stability, respectively. Each viscosity is used to identify a particular characteristic of the rice variety. Basically the starch retrogradation relatively controlled the increase in paste viscosity, whereas shear and rupturing of swollen starch granules are regulated by breakdown viscosity. Setback viscosity implies the degree of retrogradation. The study showed that usually amylographic properties are significantly influenced by abiotic factors (Martinez, 2019) ${ }^{[3]}$.

The pasting behavior of starch is a combination of complex processes that occurs after the gelatinization, which includes the starch granules change from swelling to rupture, the leaching of amylose, and the gel formation with high energy input (Zhu et al., 2020) ${ }^{[10]}$.

\section{Materials and Methods}

\subsection{Materials.}

Kodo millet sample obtained from Sri Venkateshwara Rice mill, Tirchy, Tamil Nadu, India. was used for the research. Samples for analysis were then prepared using different preconditioning methods.

\subsection{Methods}

\subsubsection{Sample preparation:}

Pre-conditioning treatments I (normal soaking): the sample was soaked in normal room temperature for 3 hours. The soaked grains were then drained which were then tempered for 1 hour. Drained grains were then steamed for 5-10 minutes which were then dried in tray drier at $45^{\circ} \mathrm{C}$ for 4 hours till m.c reduces to $11 \%$. The dried grains were then milled in Satake emery roller for separating the husk from the grains.

Pre-conditioning treatments II (Hot water soaking): the sample was soaked in hot water at $65^{\circ} \mathrm{C}$ for 2 hours in hot plate, then tempered for 1 hour after draining water. Drained grains were then steamed for 5-10 minutes which were then dried in tray drier at $45^{\circ} \mathrm{C}$ for 4 hours till m.c reduces to $11 \%$.
The dried grains were then milled in Satake emery roller for separating the husk from the grains.

Pre-conditioning treatments III (pressure parboiling): the sample was soaked in normal room temperature for half an hour then drained and pressure parboiled at $121^{\circ} \mathrm{C}$ at $15 \mathrm{psi}$ for 15 minutes. The parboiled grains were then then dried in tray drier at $45^{\circ} \mathrm{C}$ for 4 hours till m.c reduces to $11 \%$. The dried grains were then milled in Satake emery roller for separating the husk from the grain (as shown in flow diagram $1)$.

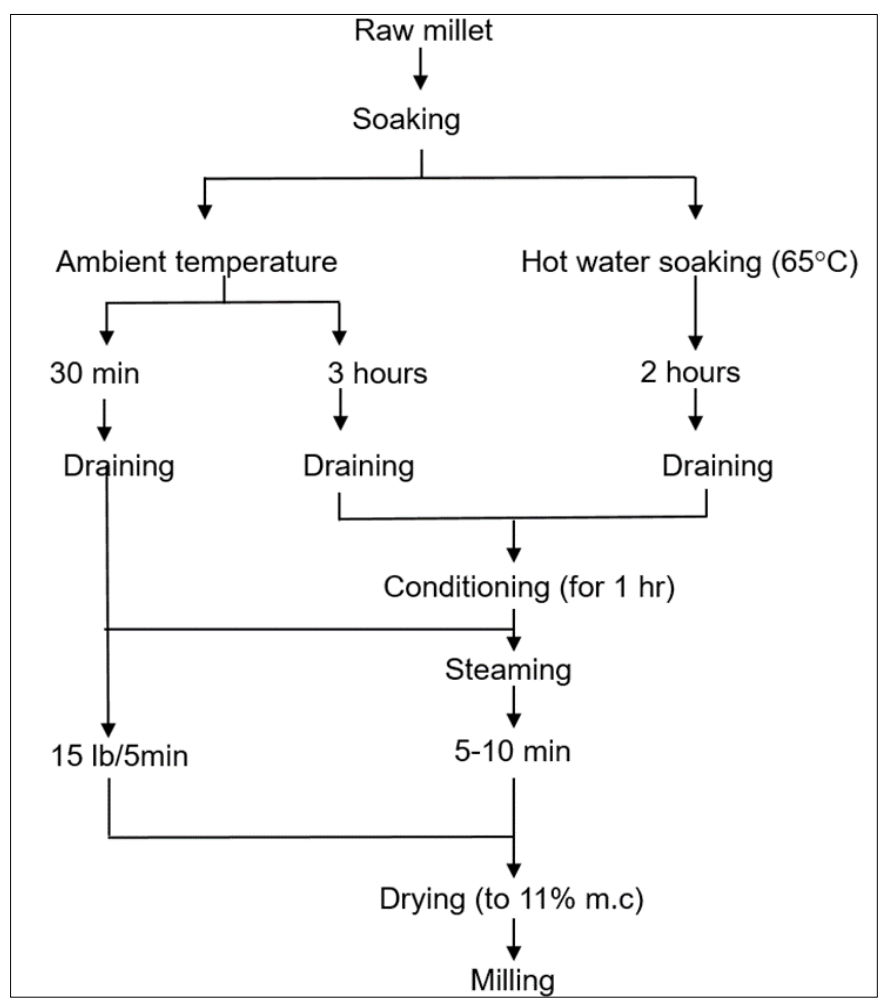

Fig 1: Flow chart of Pre-conditioning treatments.

\subsubsection{Pasting properties}

RVA The determination of pasting properties of rice cultivars was done using Rheometer (MCR 52, Anton Paar Co. Ltd.) as described by AACC (2000). A $12 \%$ (w.b) flour-water slurry was stirred in the sample canister at a rotational speed of 960 rpm for an initial $10 \mathrm{~s}$ and $160 \mathrm{rpm}$ for the rest of the procedure. The temperature-time protocol was started at $50^{\circ} \mathrm{C}$ for $1 \mathrm{~min}$ after which a linear ramp-up of temperature till $95^{\circ} \mathrm{C}$ in $3 \mathrm{~min} 42 \mathrm{~s}$ and held at that temperature for $2 \min 30 \mathrm{~s}$. After the holding time, the temperature was ramped-down to $50^{\circ} \mathrm{C}$ in $3 \mathrm{~min} 48 \mathrm{~s}$ by the cooling system. The precise heathold-cool cycle causes changes in the starch granules in samples, leading to viscosity changes. The changes in viscosity due to simultaneous heating and stirring were plotted on a curve, give the peak viscosity, final viscosity, pasting temperature and time.

\section{Result and Discussion}

The pasting properties of different treated millet grains showed varied trends in peak, trough, breakdown, final, setback viscosities and pasting time and temperature. Characteristic pasting curves with an increase of viscosity till attainment of peak after which it diminishes down during the holding period. The viscosity rises further after setback to reach final viscosity forming sharp peaks. The pasting behaviour of various starches is known to alter due to interactions between starch and non-starch components, type 
of proteins and minerals present in samples, structures and orientation of starch granules, amylose-amylopectin ratio, degree of milling, presence of impurities, $\mathrm{pH}$ and cultivars (Mahasukhonthachat et al. 2010). The greater pasting temperature was shown by control, depicting the higher energy and time required for cooking, correlating with higher amylose content. The pasting temperatures of different treatments ranged from 74.35 to $57.69{ }^{\circ} \mathrm{C}$ with $\mathrm{T} 3$ being the least and high for the control grains indicating more cooking time and energy requirement for the control and least for the T3 treated grains. Peak viscosity (PV) is an indicator of water binding capacity and ease with which the starch granules are disintegrated and often correlated with final product quality (Thomas and Atwell, 1999). The peak viscosities varied from 1,157 to $82 \mathrm{cP}$, whereas the final viscosities ranged from 3571 to $256 \mathrm{cP}$ (Table 1). Control has higher peak viscosity followed by T1, T2 and lowest for T3, indicating control has higher water binding capacity resulting in more swelling of starch granules. Final viscosity indicates the ability of the starch to form a viscous paste. A higher final viscosity relates to the high resistance to shear. Increase in final viscosity might be due to the aggregation of the amylose molecules (S.K et al., 2013) ${ }^{[7]}$. It has been reported that the higher final viscosity of the rice flours indicates greater retrogradation tendency due to the crystallization of amylose molecules that are leached from the swollen starches (Wu et al. 2011). This might be due to the amylose molecules that provide stability to starch granules against swelling and greater susceptibility for retrogradation (Singh et al. 2006). Highest final viscosity was found for the control sample followed by T1, T2 and then least for the T3. Control and T1 showed greater final viscosities showing that these two conditions hold higher resistance against the shear stress creating due to the rotation of spindle without attaining breakdown point (Falade and Christopher, 2015).

Table 1: Pasting properties of pearl millet varieties

\begin{tabular}{|c|c|c|c|c|}
\hline & Control & T1 & T2 & T3 \\
\hline Peak viscosity $(\mathrm{cP})$ & $1,157 \pm 2.82$ & $467.40 \pm 11.73$ & $157.25 \pm 13.36$ & $82.71 \pm 9.14$ \\
\hline Pasting Temperature $\left[1^{\circ} \mathrm{C}\right]$ & $74.34 \pm 0.912$ & $58.40 \pm 6.35$ & $63.07 \pm 18.53$ & $57.68 \pm 10.82$ \\
\hline Holding Viscosity $(\mathrm{cP})$ & $462.3 \pm 46.10$ & $200.25 \pm 27.50$ & $83.07 \pm 13.16$ & $48.11 \pm 15.85$ \\
\hline Breakdown Viscosity $(\mathrm{cP})$ & $694.7 \pm 85.27$ & $267.1 \pm 15.69$ & $74.16 \pm 26.53$ & $47.56 \pm 25.02$ \\
\hline Final viscosity $(\mathrm{cP})$ & $3,571 \pm 22.62$ & $1,774 \pm 45.96$ & $256.25 \pm 3.89$ & $256.02 \pm 14.70$ \\
\hline Setback from peak viscosity $(\mathrm{cP})$ & $-2,446 \pm 96.16$ & $-1,307 \pm 57.27$ & $-89.06 \pm 4.61$ & $-149.20 \pm 10.18$ \\
\hline Setback from trough viscosity $(\mathrm{cP})$ & $3459 \pm 143.54$ & $1,574 \pm 72.83$ & $173.25 \pm 17.04$ & $165.93 \pm 28.80$ \\
\hline
\end{tabular}

The breakdown is caused by disintegration of gelatinized starch granules structure during continued stirring and heating, thus, indicating the shear thinning property of starch (Yadav et al., 2011). A low breakdown value suggests the stability of starches under hot conditions. Amylose content is believed to have a marked influence on the breakdown viscosity (measure of susceptibility of cooked starch granule to disintegration) and the setback viscosity (measure of recrystallization of gelatinized starch during cooling) (Lee $e t$ al., 1995). Lower level of amylose to reinforce the molecular network within the granules resulted in greater breakdown viscosity. High amylose content has also been suggested as the major factor contributing to the non-existense of a peak, a high stability during heating, and a high setback during cooling (S.K et al., 2013) ${ }^{[7]}$. The breakdown viscosity which depicts the rate of disintegration of cooked starches is indirectly proportional to the ability of the processed sample to withstand heat and shear during cooking (Adebowale et al. 2005). The greater breakdown viscosity was found for control followed by $\mathrm{T} 1$, depicting that these treatments have a low ability to withstand shear stress and heat.

Setback viscosity from peak is an index of retrogradation, means indicating increase in viscosity resulting from the rearrangement of amylose molecules that have leached out from the swollen starch granules during cooling and is generally used as a measure of gelling ability or retrogradation tendency of starch granules. Low setback back value means it has low rate of retrogradation and hence product made of low setback value flour will have a prolonged shelf life period, here in this case, lower setback value was observed for control followed by T1, T3 and then $\mathrm{T} 2$ indicating product made with $\mathrm{T} 2$ treated grains will have lowest shelf life compared to the other treated grains. Similarly, greater trough viscosity shows the ability of formed pastes to retain viscosity without breaking during the cooling period (Nikitha \& Natarajan, 2020) ${ }^{[4]}$.

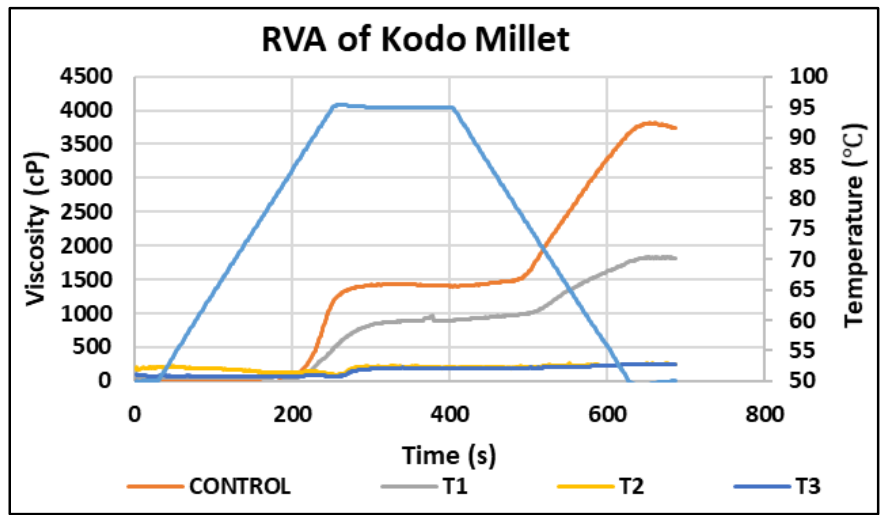

Fig 2: Viscosity profile of different treatments.

The pasting characteristics of raw and processed samples measured using a Rapid Visco Analyzer (RVA) is depicted in Fig. 2. RVA curves gave mentionable differences in pasting properties between the different treated grains. The pasting curve shapes of T2 and T3 samples were quite similar. The starch pasting profiles obtained from RVA could reflect the molecular events occurring in the starch granules during the heating cycle (Majzoobi et al., 2011). The peak viscosity indicates the ability of the granules to swell freely before breakdown (Singh et al., 2010). Rise in paste viscosity is also attributed to breaking down of long starch chains or clusters upon thermal processing which release upon cooking into the surrounding water medium. These chains remain suspended in the water, thereby increasing the viscosity of the slurry. This may be the factor behind the marginal rise in the RVA pasting curve of control sample. The increased paste viscosity in T1(Normal soaked grains) samples was however only marginally evident when compared to control ones. However, upon severity of processing treatment, the chains get further fragmented and modified to such an extent, where the granules are not able to hold themselves in the slurry and 
sediment to decrease the viscosity. The Hot water soaked (T2) and Pressure parboiled (T3) samples therefore exhibited lowering of viscosity, which can be due to the extensive starch breakdown and therefore marked lowering of the viscosities, similar result was observed while studying the effect of hydrothermal processing techniques on quality of Buckwheat grains and flour.

\section{Conclusion}

The result of pasting property showed that the starch suspension viscosity of hot soaked (T2) and pressure parboiled (T3) samples decreased severely compared to raw control millet sample due to extensive starch breakdown. Whereas in case of normal soaked (T1) grains, the changes were moderate in comparison to the control sample.

\section{References}

1. De Wet JMJ, Brink DE, Rao KEP, Mengesha MH. Diversity in Kodo millet, Paspalum scrobiculatum. Economic Botany 1983;37(2):159-163. https://doi.org/10.1007/BF02858779

2. Kweon M, Slade L, Levine H, Souza E. Application of RVA and Time-Lapse photography to explore effects of extent of chlorination, milling extraction rate, and particle-size reduction of flour on cake-baking functionality. Cereal Chemistry 2010;87(5):409-414. https://doi.org/10.1094/CCHEM-03-10-0046

3. Martinez MM. Applications of the Rapid Visco Analyser (RVA) in the Food Industry: a broader view. Perten Instruments, January 2019, 14-21. https://www.perten.com/Publications/Articles/Applicatio ns-of-the-Rapid-Visco-Analyser-RVA-in-the-FoodIndustry-a-broader-view/

4. Nikitha M, Natarajan V. Properties of South-Indian rice cultivars: physicochemical, functional, thermal and cooking characterisation. Journal of Food Science and Technology 2020. https://doi.org/10.1007/s13197-02004440-3

5. Rapid Visco Analyser 2000.

6. Rathore S. Millet Grain Processing, Utilization and Its Role in Health Promotion: A Review. International Journal of Nutrition and Food Sciences 2016;5(5):318. https://doi.org/10.11648/j.ijnfs.20160505.12

7. SK B BR, SY B. Physicochemical, functional, thermal and pasting properties of starches isolated from pearl millet cultivars 2013;20(4):1555-1561.

8. Shahidi F, Chandrasekara A. Millet grain phenolics and their role in disease risk reduction and health promotion: A review. Journal of Functional Foods 2013;5(2):570581. https://doi.org/10.1016/j.jff.2013.02.004

9. Spengler RN. The Millets. In Fruit from the Sands 2019, pp 59-88. https://doi.org/10.2307/j.ctvh1dx4s.9

10. Zhu L, Zhang Y, Wu G, Qi X, Dag D, Kong F, Zhang H. International Journal of Biological Macromolecules Characteristics of pasting properties and morphology changes of rice starch and fl our under different heating modes. International Journal of Biological Macromolecules 2020;149:246-255. https://doi.org/10.1016/j.ijbiomac.2020.01.161 